CONFORMAL GEOMETRY AND DYNAMICS

An Electronic Journal of the American Mathematical Society

Volume 2, Pages 89-106 (October 14, 1998)

S $1088-4173(98) 00032-0$

\title{
MÖBIUS INVARIANT QUATERNION GEOMETRY
}

\author{
R. MICHAEL PORTER
}

\begin{abstract}
A covariant derivative is defined on the one point compactification of the quaternions, respecting the natural action of quaternionic Möbius transformations. The self-parallel curves (analogues of geodesics) in this geometry are the loxodromes. Contrasts between quaternionic and complex Möbius geometries are noted.
\end{abstract}

\section{INTRODUCTION}

Let $\mathbf{H}$ denote the skew field of quaternions, and $\widehat{\mathbf{H}}=\mathbf{H} \cup\{\infty\}$ the compactification to the 4-sphere. Consider a smooth curve $\gamma$ in $\widehat{\mathbf{H}}$. We define a "covariant derivative" $D_{\gamma}$ along $\gamma$ which is invariant under the full quaternionic Möbius group Aut $\widehat{\mathbf{H}}$. The construction goes along the following lines: A loxodrome is any image under an element of Aut $\widehat{\mathbf{H}}$ of a spiral $t \mapsto e^{t u} q e^{-t v}$, where $q, u, v \in \mathbf{H}$. There is a fiber bundle $\pi_{\mathcal{B}}: \mathcal{B} \rightarrow \widehat{\mathbf{H}}$ of all germs of loxodromes, where the germ $\beta \in \mathcal{B}$ is based at $\pi_{\mathcal{B}}(\beta)$. Suppose $t \mapsto \beta_{t}$ is a curve in $\mathcal{B}$ lying over $\gamma$, that is, $\pi_{\mathcal{B}}\left(\beta_{t}\right)=\gamma(t)$. Then $D_{\gamma} \beta$, the covariant derivative of this loxodromic germ field on $\gamma$, is defined generically to lie in $\mathcal{B}$, and for any $T \in \operatorname{Aut} \widehat{\mathbf{H}}, D_{T \circ \gamma}\left(T_{*}(\beta)\right)=T_{*}\left(D_{\gamma} \beta\right)$. Because of this invariance, $D_{\gamma}$ is also well defined for a curve $\gamma$ in a manifold modeled on (Aut $\widehat{\mathbf{H}}, \widehat{\mathbf{H}})$.

An analogous construction was carried out in [19] for the Riemann sphere $\widehat{\mathbf{C}}$ in place of $\widehat{\mathbf{H}}$. The quaternionic context presents numerous technical questions (in particular, how to "divide" one element of the Lie algebra by another), which are treated in Sections 3 through 5.

There are many "Möbius groups" provided in the literature in the context of Clifford algebras, especially via Clifford-Vahlen matrices. The group Aut $\widehat{\mathbf{H}}$ considered here and in [10], [21] does not coincide precisely with the Möbius groups used, for example, by Ahlfors [1] and others, which leave a half-space invariant and have an invariant hyperbolic metric.

We will require a rather large quantity of preliminary definitions and results. To make this paper self contained, the bundle $\mathcal{B}$ and its natural flow are defined in Section 2. Section 3 is concerned with the fixed points of elements of Aut $\widehat{\mathbf{H}}$ and their smooth dependence on parameters. Section 4 describes in detail the structure of hyperbolic elements, in particular those which we call "strongly hyperbolic".

Received by the editors January 29, 1998 and, in revised form, August 25, 1998.

1991 Mathematics Subject Classification. Primary 53A55; Secondary 53B10, 15A66, 51N30, 20G20.

Key words and phrases. Quaternion, Möbius transformation, loxodrome, covariant derivative. Partially supported by CONACyT grant 211085-5-2585P-E.

(C)1998 American Mathematical Society 
The 5-jets which correspond to loxodromic trajectories are calculated in Section 5 . The covariant derivative and the parallel transport which it determines are finally defined in Section 6. Some additional observations are reserved for the final section.

This work is dedicated to the memory of Peter Greenberg, who as well as being a true friend had the original idea leading to the definition of the flow-bundle $\mathcal{B}$.

\section{NotATION AND DEFINITIONS}

2.1. Quaternions. We work with the set of (real) quaternions $\mathbf{H}=\left\{q=q_{0}+q_{1} i+\right.$ $\left.q_{2} j+q_{3} k: q_{\alpha} \in \mathbf{R}\right\}$; the nonreal generators $i, j, k$ anticommute and their squares are -1 . The norm $|q|^{2}=q \bar{q}=q_{0}^{2}+q_{1}^{2}+q_{2}^{2}+q_{3}^{2}$ is the Euclidean norm with the natural identification of $\mathbf{H}$ with $\mathbf{R}^{4}$.

Write $\mathbf{C}=\left\{q_{0}+q_{1} i \in \mathbf{H}\right\}$; the other isomorphic embeddings of the complex numbers in $\mathbf{H}$, equally natural, are given by $\mathbf{R}+\mathbf{R} \omega, \omega \in S^{2}=\{q \in \mathbf{H} ;|q|=$ $1, \operatorname{Re} q=0\}$. Here $\omega$ plays the role of the imaginary unit $i$. Sometimes it will be convenient to use the orthogonal decomposition $\mathbf{H}=\mathbf{C} \oplus \mathbf{C} j$. For more details on the elementary properties of quaternions one may consult [9], [13] and the references there.

2.2. Quaternionic Möbius transformations. We will denote by $\mathbf{H}^{2 \times 2}$ the set of 2-by-2 matrices with entries in $\mathbf{H}$. An element

$$
A=\left(\begin{array}{ll}
a_{00} & a_{01} \\
a_{10} & a_{11}
\end{array}\right)
$$

will also be written sometimes as $A=\left(a_{m n}\right)$ or $A=\left(a_{00}, a_{01} ; a_{10}, a_{11}\right)$. Matrix multiplication is defined in the usual way, i.e., $A A^{\prime}=\left(a_{m 0} a_{0 n}^{\prime}+a_{m 1} a_{1 n}^{\prime}\right)$. The right-linear mappings $L_{A}$ of $\mathbf{H}^{2}$ given by $L_{A}(V)=A V$ satisfy $L_{A A^{\prime}}=L_{A} L_{A^{\prime}}$. Write $\mathrm{GL}_{2} \mathbf{H} \subseteq \mathbf{H}^{2 \times 2}$ for the subset of invertible matrices; it is well known [3], [9] that $A$ has a two-sided inverse in $\mathbf{H}^{2 \times 2}$ if and only if the real-linear mapping induced by $L_{A}$ on $\mathbf{R}^{8}$ is invertible.

For $A \in \mathrm{GL}_{2} \mathbf{H}$ we define the right-Möbius transformation

$$
F_{A}(q)=\left(a_{00} q+a_{01}\right)\left(a_{10} q+a_{11}\right)^{-1}
$$

it is easily seen that $F_{A A^{\prime}}=F_{A} \circ F_{A^{\prime}}$ with the usual conventions $F_{A}(\infty)=a_{00} a_{10}^{-1}$, $F_{A}\left(-a_{11} a_{10}^{-1}\right)=\infty$. Thus $F_{A}$ is a self mapping of the 4 -sphere $\widehat{\mathbf{H}}=\mathbf{H} \cup\{\infty\}$, and is of class $C^{\infty}$ when $\mathbf{H}$ is given the obvious differentiable structure under which the inversion $q \mapsto q^{-1}$ is smooth.

As is the case with $\widehat{\mathbf{C}}$, there is a Möbius transformation taking any three given points to any other three points; however, it is not unique. Define

$$
\text { Aut } \widehat{\mathbf{H}}=\left\{F_{A}: A \in \mathrm{GL}_{2} \mathbf{H}\right\} \text {. }
$$

This set is identified naturally with the quotient spaces $\mathrm{PGL}_{2} \mathbf{H}=\mathrm{GL}_{2} \mathbf{H} / \mathbf{R}=$ $\mathrm{SL}_{2} \mathbf{H} /( \pm I)$, where

$$
\mathrm{SL}_{2} \mathbf{H}=\left\{A \in \mathrm{GL}_{2} \mathbf{H}: \operatorname{det}_{\mathbf{R}} A=1\right\},
$$

$\operatorname{det}_{\mathbf{R}} A$ denoting the determinant of the real-linear map on $\mathbf{R}^{8}$ induced by $L_{A}$. From now on, for $A \in \mathrm{SL}_{2} \mathbf{H}$ we will freely write $A$ in place of $F_{A} \in \operatorname{Aut} \widehat{\mathbf{H}}$ when there is no danger of confusion.

It is well known (see, for example [21]) that the class of left-Möbius transformations $(q c+d)^{-1}(q a+b)$ coincides with Aut $\widehat{\mathbf{H}}$. 


\subsection{Exponential mapping and one-parameter families. Write}

$$
\operatorname{sl}_{2} \mathbf{H}=\left\{X \in \mathbf{H}^{2 \times 2}: \operatorname{Retr} X=0\right\}=\mathbf{H}^{2 \times 2} \cap \mathrm{sl}_{8} \mathbf{R},
$$

where $\operatorname{tr} X=x_{00}+x_{11} \in \mathbf{H}$. This is a sub-Lie algebra of $\operatorname{sl}_{8} \mathbf{R}$ with the usual bracket operation $X Y-Y X$. The exponential series

$$
e^{X}=\exp (X)=\sum_{0}^{\infty} \frac{1}{n !} X^{n}
$$

converges for all $X \in \mathbf{H}^{2 \times 2}$. Note that $X \in \operatorname{sl}_{2} \mathbf{H}$ implies $\operatorname{det} \mathbf{R} \exp (X)=1$. Thus $\exp : \operatorname{sl}_{2} \mathbf{H} \rightarrow$ Aut $\widehat{\mathbf{H}}$ is induced by $\exp : \mathrm{sl}_{2} \mathbf{H} \rightarrow \mathrm{SL}_{2} \mathbf{H}$. We will make frequent use of the adjoint representation

$$
\operatorname{Ad} T: \mathrm{sl}_{2} \mathbf{H} \rightarrow \mathrm{sl}_{2} \mathbf{H}, \quad \operatorname{Ad} T(X)=T X T^{-1},
$$

which satisfies $e^{\operatorname{Ad} T(X)}=T e^{X} T^{-1}$. Further details may be found in [21].

\section{The NATURAL QUATERniOniC FLOW}

3.1. Loxodromes. The one-parameter family $t \mapsto \exp (t X)$ of $X \in \mathrm{sl}_{2} \mathbf{H}$ determines a flow on $\mathbf{H}$ in a natural way. Given $q \in \widehat{\mathbf{H}}$, the $X$-loxodrome based at $q$ is the trajectory

$$
\sigma(t)=e^{t X}(q)
$$

If $X=0$, or more generally, if $q$ is a common fixed point of the family $\left\{e^{i t}\right\}$ (cf. Proposition 4.3 below), then $\sigma$ is constant; otherwise it is a nonsingular $C^{\infty}$ curve (this dichotomy follows from Proposition 6.1). It was shown in [21] that a loxodrome is the same as the image of a quaternionic spiral $t \mapsto e^{t u} q e^{-t v}(q, u, v \in$ H) under a Möbius transformation. A loxodrome is planar if it lies in some 2-plane through the origin; such loxodromes reduce essentially to loxodromes in $\mathbf{C}$. We cite the following, which shows that $\mathrm{sl}_{2} \mathbf{H}$ is the appropriate space for parametrizing loxodromes.

Proposition 3.1 ([21]). Let $X, Y \in \mathrm{sl}_{2} \mathbf{H}$ generate the same nonplanar loxodrome; that is, suppose $e^{t X}(\infty)=e^{t Y}(\infty)$ for all $t \in \mathbf{R}$. Then $X=Y$.

In order to compute with germs of loxodromes, we will follow quite closely the notation used in [19] for the complex Möbius group Aut $\widehat{\mathbf{C}}$. A point $q \in \widehat{\mathbf{H}}$ can be represented by any transformation $A \in$ Aut $\widehat{\mathbf{H}}$ such that $A(\infty)=q$. Thus there is a natural identification $\widehat{\mathbf{H}} \cong$ Aut $\widehat{\mathbf{H}} /$ Aff $\mathbf{H}$, where

$$
\text { Aff } \mathbf{H}=\{T \in \text { Aut } \widehat{\mathbf{H}}: T(\infty)=\infty\}
$$

is the quaternionic affine group; it consists of those Möbius transformations represented by upper triangular matrices. We will write $[A]$ for the equivalence class of $A$, thus $[A]=[A T]$ for $T \in$ Aff $\mathbf{H}$. The main object of study in this paper is the quotient bundle

$$
\begin{aligned}
\mathcal{B} & =\left(\text { Aut } \widehat{\mathbf{H}} \times \mathrm{sl}_{2} \mathbf{H}\right) / \text { Aff } \mathbf{H}, \\
{[A, X] } & =\left[A T^{-1}, \operatorname{Ad} T(X)\right], T \in \operatorname{Aff} \mathbf{H} .
\end{aligned}
$$

An element $\alpha=[A, X]$ of $\mathcal{B}$ represents both a point $q=A(\infty)$ and a loxodromic trajectory $\exp (t X)(q)$ based at $q$. We may also think of $\alpha$ as representing the germ 
of the trajectory based at $q$; note that all representatives for $\alpha$ determine the same trajectory. There is a natural projection $\pi_{\mathcal{B}}: \mathcal{B} \rightarrow \widehat{\mathbf{H}}$ defined by

$$
\pi_{\mathcal{B}}([A, X])=A(\infty) .
$$

The fiber $\pi_{\mathcal{B}}^{-1}(q)$ represents all loxodromes based at $q$. The natural flow $\Phi_{\mathcal{B}}: \mathcal{B} \times$ $\mathbf{R} \rightarrow \mathcal{B}$ is defined by

$$
\Phi_{\mathcal{B}}(\alpha, t)=\left[A e^{t X}, X\right] \quad(\alpha=[A, X] \in \mathcal{B}, \quad t \in \mathbf{R}) .
$$

Thus $\Phi_{\mathcal{B}}(\alpha, t)$ represents the same loxodrome $\alpha$, with its base point moved along the loxodrome corresponding to time $t$. This is analogous to the geodesic flow on the tangent bundle of a Riemannian manifold, although in the present context there is no metric invariant under the corresponding group (see [7]).

Given $\alpha \in \mathcal{B}$, the flow $\Phi_{\mathcal{B}}$ projects via $\pi_{\mathcal{B}}$ to the natural flow $\varphi_{\mathcal{B}}$ on $\widehat{\mathbf{H}}$ determined by $\alpha$ as follows: since $\pi_{\mathcal{B}}\left(\Phi_{\mathcal{B}}(\alpha, t)\right)=A(\exp (t X)(\infty))$ is the associated loxodrome passing through $\pi_{B}(\alpha)$, we write, for any $q$,

$$
\varphi_{\mathcal{B}}(\alpha, t)(q)=A\left(e^{t X}(q)\right)
$$

which is a loxodromic trajectory. Thus $\pi_{\mathcal{B}} \Phi_{\mathcal{B}}(\alpha, t)$ is just one leaf of the flow on $\widehat{\mathbf{H}}$ determined by $\alpha$.

This construction can be carried over to manifolds modeled on (Aut $\widehat{\mathbf{H}}, \widehat{\mathbf{H}}$ ). In other words, let $M$ be a 4-dimensional real manifold with coordinate charts taking values in $\mathbf{H}$, such that all of the coordinate transition functions are restrictions of elements of Aut $\widehat{\mathbf{H}}$. Then the restrictions of $\mathcal{B}$ to the coordinate images can be pieced together in a natural way to form a bundle $\mathcal{B}(M) \rightarrow M$. The flow $\Phi_{\mathcal{B}}$ on $\mathcal{B}$ transfers to a local flow on $\mathcal{B}(M)$; its trajectories project to (locally) loxodromic curves on $M$. The analogous structures of type (Aut $\widehat{\mathbf{C}}, \widehat{\mathbf{C}})$, commonly known as projective structures, have an ample literature; see for instance [8], [14], [15].

The covariant derivative defined in Section 6 will be invariant under Möbius transformations, and thus will live on such quaternionic Möbius manifolds.

\section{Classification of elements of Aut $\widehat{\mathbf{H}}$}

For $\lambda \in \mathbf{H}-\{0\}$, write

$$
C_{\lambda}(q)=\lambda q \lambda^{-1}
$$

we say $q$ is similar to $C_{\lambda}(q)$. The following classification by similarity is well known.

Lemma 4.1. Let $p, q \in \mathbf{H}$. Then $p$ is similar to $q$ in $\mathbf{H}$ if and only if $|p|=|q|$ and $\operatorname{Re} p=\operatorname{Re} q$. Let $\lambda \in \mathbf{H}-\{0\}$. Then $C_{\lambda}(i) \in \mathbf{C}$ if and only if $\lambda \in \mathbf{C} \cup \mathbf{C} j$. For $\lambda \in \mathbf{C}, C_{\lambda}$ acts as the identity on $\mathbf{C}$, while for $\lambda \in \mathbf{C} j$ it acts as complex conjugation on $\mathbf{C}$.

From the lemma, if $q$ is a pure quaternion $(\operatorname{Re} q=0)$, then so is $C_{\lambda}(q)$. If $C_{\lambda}$ is the identity on $\mathbf{H}$, then $\lambda \in \mathbf{R}$; that is, the purely nonreal part of $\lambda$ is zero.

4.1. Fixed points. Let $A \in$ Aut $\widehat{\mathbf{H}}$. From (2.1), the fixed point set of $A$ can be described as

$$
\operatorname{Fix} A=\left\{q \in \mathbf{H}: P_{A}(q)=0\right\}
$$


where for any $X=\left(x_{00}, x_{01} ; x_{10}, x_{11}\right) \in \mathbf{H}^{2 \times 2}$, we write

$$
P_{X}(q)=-q x_{10} q-q x_{11}+x_{00} q+x_{01} .
$$

By convention we say $P_{X}(\infty)=0$ when $x_{10}=0$. It is clear that $P_{X}(q)=0$ for all $q$ if and only if $X$ is a real multiple of the identity matrix $I$.

Due to the importance of the quaternionic quadratic polynomial $P_{X}$ in what follows, we will examine closely certain aspects which will be relevant to Möbius transformations. First consider the linear function to which $P_{X}$ reduces when $x_{10}=0$. The mapping

$$
q \mapsto-q x_{11}+x_{00} q
$$

has as kernel $\{0\} \cup\left\{q: C_{q}\left(x_{11}\right)=x_{00}\right\}$. This kernel is trivial unless $x_{00}$ and $x_{11}$ are similar in $\mathbf{H}$; if it is not trivial, then it has real dimension 2 unless $x_{00}$ and $x_{11}$ are real and equal, in which case it is all of $\mathbf{H}$. An explicit solution to $-q x_{11}+x_{00} q=$ $-x_{01}$ is written out in [20] for the case that $x_{00}, x_{11}$ are not similar.

In general there is no explicit solution available for the quadratic equation $P_{X}(q)=0$. At least one zero exists by topological reasons [18]. If one zero $q$ is known, the nature of the solution set can then be determined explicitly, according to the following result.

Proposition 4.2 ([10], [20]). Let $X \in \mathbf{H}^{2 \times 2}$. The set of zeroes in $\widehat{\mathbf{H}}$ of the quadratic polynomial $P_{X}$ given by (4.2) consists of one point, of two points, of a Euclidean 2-sphere in $\widehat{\mathbf{H}}$, or all of $\widehat{\mathbf{H}}$. Suppose $X$ is not a real multiple of $I$. Given $q \neq \infty$ such that $P_{X}(q)=0$, the set of zeroes of $P_{X}$ is a 2-sphere if and only if $x_{10} q+x_{11}$ is similar to $-q x_{10}+x_{01}$ in $\mathbf{H}$.

As is customary in Möbius geometry, we regard an affine 2-plane together with $\infty$ as a special case of a 2 -sphere. For $A \in \mathrm{GL}_{2} \mathbf{H}-\{I\}$ we will say that $A \in$ Aut $\widehat{\mathbf{H}}$ is parabolic, hyperbolic, or elliptic if it fixes one, two, or infinitely many points.

The following basic fact was proved in [21].

Proposition 4.3. Let $X \in \mathrm{sl}_{2} \mathbf{H}$ and let $q \in \widehat{\mathbf{H}}$. Then $q$ is a fixed point of the entire one-parameter family $\left\{e^{t X}\right\} \subseteq$ Aut $\widehat{\mathbf{H}}$ if and only if $P_{X}(q)=0$.

Because of this, for convenience we may refer to the solutions of $P_{X}(q)=0$ as the "fixed points" of $X \in \mathrm{sl}_{2} \mathbf{H}$. From Proposition 4.3 it follows that the upper triangular elements of $\mathrm{sl}_{2} \mathbf{H}$ are precisely those for which the associated one-parameter family fixes the point at infinity; we will call these affine matrices (even though not all such are invertible). Similarly the diagonal matrices are those whose oneparameter families fix $0, \infty$. We define the type of $X \in \mathrm{sl}_{2} \mathbf{H}-\{0\}$ (i.e., parabolic, hyperbolic, or elliptic) to be the type of the one-parameter family $\left\{e^{t X}\right\}$ that it generates.

Corollary 4.4. $X \in \mathrm{sl}_{2} \mathbf{H}-\{0\}$ is parabolic, hyperbolic, or elliptic according to whether the quadratic polynomial $P_{X}$ has one, two, or infinitely many zeros.

We will be interested principally in hyperbolic transformations. In [20] it was shown that $P_{X}(q)=0$ has exactly two solutions for a dense set of coefficients $X \in \mathbf{H}^{2 \times 2}$, and it was conjectured that this set is also open. This will follow from Lemma 4.6 below.

If $A$ fixes the points $q, q^{\prime}$, and if $T$ takes $\left(q, q^{\prime}\right)$ to $(0, \infty)$, then $D=T A T^{-1}$ is of the form $\left(d_{00}, 0 ; 0, d_{11}\right)$, and the condition $\operatorname{det}_{\mathbf{R}} D=1$ can be seen to imply 
$\left|d_{00} d_{11}\right|=1$. This diagonalization is examined in more detail in Section 4. One sees from Proposition 4.2 that $A$ is elliptic if and only if $d_{00}$ is similar to $d_{11}$; this condition obviously does not depend on the diagonalizing transformation $T$.

4.2. Fixed points as a function of the matrix. It will be important for us to know that the fixed points of a hyperbolic one-parameter family depend smoothly on the entries of its generator. We devote the rest of this section to this point.

In [21] it was observed that each element $A \in$ Aut $\widehat{\mathbf{H}}-$ Aff $\mathbf{H}$ can be expressed as a composition of an orthogonal mapping, an inversion in a sphere (the isometric sphere) and a reflection in a plane, analogously to the well known decomposition for elements of Aut $\widehat{\mathbf{C}}$ [4]. Further, these elementary transformations can be written down explicitly as algebraic functions of the entries of $A$. The mapping $A$ is parabolic when its isometric spheres are tangent and the orthogonal mapping is the identity. As a consequence of these facts we have the following.

Lemma 4.5. The set $\{A \in$ Aut $\widehat{\mathbf{H}}: A$ is parabolic $\} \cup\{I\}$ is a closed set.

We now show that the set of hyperbolic elements is open.

Lemma 4.6. Let $A \in$ Aut $\widehat{\mathbf{H}}$ be hyperbolic and let $\left\{A_{n}\right\}$ be a sequence converging to $A$. Let $q_{n} \in$ Fix $A_{n}$. Then the sequence $\left\{q_{n}\right\}$ accumulates only to the fixed points of $A$. Further, for sufficiently large $n, A_{n}$ is hyperbolic.

Proof. We may assume $A=\left(a_{00}, 0 ; 0, a_{11}\right)$ is diagonal. From Proposition 4.3 we have $P_{A_{n}}\left(q_{n}\right)=0$. Since $a_{10}^{(n)} \rightarrow a_{10}=0, a_{01}^{(n)} \rightarrow a_{01}=0$ as $n \rightarrow \infty$, it follows from (4.2) that $q_{n} a_{11}^{(n)}-a_{00}^{(n)} q_{n} \rightarrow 0$. Now suppose the first assertion of the lemma is false; that is, on some subsequence $q_{n} \rightarrow q^{*} \neq 0, \infty$. Then $q_{n}^{-1} \rightarrow\left(q^{*}\right)^{-1}$, so $a_{00}=C_{q^{*}}\left(a_{11}\right)$. By Proposition 4.2, this implies that $A$ is elliptic, contrary to hypothesis, so in fact $\left\{q_{n}\right\}$ may not accumulate anywhere but to $0, \infty$. This proves the first assertion.

To prove the second assertion, we will assume instead that the two fixed points of $A$ are finite. Write

$$
p_{n}=a_{10}^{(n)} q_{n}+a_{11}^{(n)}, \quad p_{n}^{\prime}=-q_{n} a_{10}^{(n)}+a_{01}^{(n)} .
$$

Suppose that (on a subsequence) $A_{n}$ is nonhyperbolic; by Lemma 4.5 we may assume $A_{n}$ is elliptic. Then $p_{n}, p_{n}^{\prime}$ are similar, so by Lemma $4.1, \operatorname{Re} p_{n}=\operatorname{Re} p_{n}^{\prime}$, $\left|p_{n}\right|=\left|p_{n}^{\prime}\right|$. We have already seen that the fixed points of $A_{n}$ may only accumulate to those of $A$; say $q_{n} \rightarrow q$. Since $A_{n} \rightarrow A$ we have that $p_{n} \rightarrow p, p_{n}^{\prime} \rightarrow p^{\prime}$ where $p=a_{10} q+a_{11}, p^{\prime}=-q a_{10}+a_{01}$. Then $\operatorname{Re} p=\operatorname{Re} p^{\prime},|p|=\left|p^{\prime}\right|$; i.e., $p$ is similar to $p^{\prime}$. By Proposition 4.2, $A$ is elliptic, contrary to hypothesis. Therefore a sequence of nonhyperbolic elements cannot converge to $A$.

Theorem 4.7. Let $\left\{A_{t}\right\} \subseteq$ Aut $\widehat{\mathbf{H}}$ be a hyperbolic family varying smoothly with respect to the real parameter $t$, and let $q_{t}$ be a fixed point of $A_{t}$ varying continuously with $t$. Then $q_{t}$ depends smoothly on $t$.

Proof. We may replace $A_{t}$ by $T A_{t} T^{-1}$ and $q_{t}$ with $T\left(q_{t}\right)$ if necessary, so that $q_{0}=0$. Define $F:$ Aut $\widehat{\mathbf{H}} \times \mathbf{H} \rightarrow \mathbf{H}$ by

$$
F(A, q)=P_{A}(q)
$$

which is $C^{\infty}$-smooth. We will work in a neighborhood of $\left(A_{0}, 0\right)$. From (4.2),

$$
\frac{d}{d t} F\left(A_{t}, q_{t}\right)=\frac{d}{d t}\left(-q\left(a_{10} q+a_{11}\right)+\left(a_{00} q+a_{01}\right)\right)
$$


the derivative at $t=0$ is $-\dot{q} a_{11}+a_{00} \dot{q}+\dot{a}_{01}$. Therefore the Jacobian of $F$, restricted to the second variable, is the linear map $\dot{q} \mapsto-\dot{q} a_{11}+a_{00} \dot{q}$. Since the hyperbolicity hypothesis implies that $a_{00}$ is not similar to $a_{11}$, this is nonsingular as a map in $\mathbf{R}^{4}$. By the Implicit Function Theorem, $q_{t}$ is of class $C^{1}$ assuming that $A_{t}$ is. The higher derivatives exist because of the nonsingularity of this same map: write

$$
F_{1}(A, \dot{q})=\frac{\partial}{\partial t} F\left(A_{t}, q_{t}\right)
$$

that is, expand the right-hand side by the Chain Rule and consider the result to be a function $F_{1}$ : Aut $\widehat{\mathbf{H}} \times \mathbf{H} \rightarrow \mathbf{H}$ of the dummy variables $A, \dot{q}$. When this is done, it is seen that at $t=0$,

$$
\frac{\partial F_{1}(A, \dot{q})}{\partial \dot{q}}=-\dot{q} a_{11}+a_{00} \dot{q},
$$

and the nonsingularity implies that $\dot{q}_{t}$ is a smooth function of $A_{t}$; i.e., the second derivative $\ddot{q}$ exists. The argument may be repeated as many times as $A_{t}$ has continuous derivatives.

Theorem 4.7 may also be seen from the general theory of stability of hyperbolic sets, as in [23].

\section{INVARIANT VELOCITY OF STRONGLY HYPERBOLIC GENERATORS}

5.1. Strongly hyperbolic transformations. Recalling the discussion prior to Lemma 4.6, let $T A T^{-1}=\left(d_{00}, 0 ; 0, d_{11}\right)$ be diagonal. If $\left|d_{00}\right|>\left|d_{11}\right|$, the attractive fixed point is $\infty$. If $\left|d_{00}\right|<\left|d_{11}\right|$, then $T^{\prime} A T^{\prime-1}$ is as described in the previous sentence when $T^{\prime}=(0,-1 ; 1,0)$. If $\left|d_{00}\right|=\left|d_{11}\right|$, it is still possible for $A$ to be hyperbolic, but the fixed points are neither attracting nor repelling. We will say that $A$ is strongly hyperbolic when $\left|d_{00}\right| \neq\left|d_{11}\right|$. This property is independent of the diagonalizing transformation $T$. Similarly, $X \in \mathrm{sl}_{2} \mathbf{H}$ is called strongly hyperbolic if the family $e^{t X}$ is. We say that $T$ properly diagonalizes $A$ or $X$ if it takes the ordered pair $\left(q_{-}, q_{+}\right)$of repelling, attracting fixed points to $(0, \infty)$; such a $T$ is not unique.

Suppose $A$ is strongly hyperbolic. By Lemma 4.6 , any $A^{\prime}$ sufficiently near $A$ is hyperbolic and has fixed points near those of $A$. Therefore we may take $T^{\prime}$ near $T$ so that $D^{\prime}=T^{\prime} A^{\prime} T^{\prime-1}$ is also diagonal, and this implies that $D^{\prime}$ is near $D$. Therefore $A^{\prime}$ is also strongly hyperbolic. The open set of strongly hyperbolic transformations is also dense: it was shown in [20] that the hyperbolic elements are dense, and each of these may be perturbed arbitrarily slightly to a strongly hyperbolic element.

The following is a consequence of Lemma 4.6, Theorem 4.7, and the definition of strongly hyperbolic.

Proposition 5.1. Let $X_{t} \in \mathrm{sl}_{2} \mathbf{H}$ depend smoothly on $t \in(-\varepsilon, \varepsilon)$, and assume that $X_{t}$ is strongly hyperbolic for all $t$. Then the attractive and repelling fixed points of $X_{t}$ also depend smoothly on $t$.

5.2. Invariant velocity. If $A \in$ Aut $\widehat{\mathbf{H}}$ is hyperbolic, then it can obviously be diagonalized. By Proposition 4.3 the same holds for hyperbolic $X \in \mathrm{sl}_{2} \mathbf{H}$. It is intuitively clear that the size of the elements of the diagonal matrix should reflect in some way the rate of growth of $\exp (t X)$, and thus affect the velocity of a loxodromic 
trajectory with the generator $X$. In this subsection we will define the velocity $v(X)$, which will enjoy the invariance property $v(X)=v(\operatorname{Ad} T(X))$.

Given $v=\left(v_{0}, v_{1}, v_{2}\right) \in \mathbf{R}^{3}$, we form the diagonal matrix

$$
D(v)=\left(\begin{array}{cc}
v_{0}+v_{1} i & 0 \\
0 & -v_{0}+v_{2} i
\end{array}\right) \in \mathrm{sl}_{2} \mathbf{H} .
$$

Lemma 5.2. Let $v, v^{\prime} \in \mathbf{R}^{3}$. If there exists $\Lambda \in$ Aut $\widehat{\mathbf{H}}$ which fixes $0, \infty$ such that $\operatorname{Ad} \Lambda(D(v))=D\left(v^{\prime}\right)$, then $v_{0}^{\prime}=v_{0}, v_{1}^{\prime}= \pm v_{1}, v_{2}^{\prime}= \pm v_{2}$.

Proof. Assume that $v \neq(0,0,0)$, since otherwise the statement is trivial. By hypothesis, $\Lambda=\left(\lambda_{00}, 0 ; 0, \lambda_{11}\right)$ must be diagonal. By Lemma 4.1 it follows that $\lambda_{00}, \lambda_{11} \in \mathbf{C} \cup \mathbf{C} j$ (recall the beginning of Section 2), so the elements of the main diagonal of $D(v)$ are either equal or conjugate to the corresponding elements of $D\left(v^{\prime}\right)$.

(A typical diagonal matrix in $\operatorname{sl}_{2} \mathbf{H}$ is of the form $\left(v_{0}+v_{1} \omega, 0 ; 0,-v_{0}+v_{2} \omega^{\prime}\right)$ where $\omega, \omega^{\prime} \in S^{2}$ and $v_{0}, v_{1}, v_{2}$ are real. By Lemma 4.1 every such matrix is similar via a diagonal matrix to some $D(v)$. As a result, nothing would be gained for our purposes in considering general $\omega, \omega^{\prime}$ instead of $i$ in (5.1).)

Definition 5.3. For $c=\left(c_{0}, c_{1}, c_{2}\right) \in \mathbf{R}^{3}$ and $v \in \mathbf{R}^{3}$, we write

$$
c D(v)=D(c v)
$$

where $c v=\left(c_{0} v_{0}, c_{1} v_{1}, c_{2} v_{2}\right)$.

Definition 5.4. Let $X \in \mathrm{sl}_{2} \mathbf{H}$ be strongly hyperbolic and let $T \in$ Aut $\widehat{\mathbf{H}}$ properly diagonalize $X, \operatorname{Ad} T(X)=D(v)$. We define the invariant velocity $v(X)=$ $\left(v_{0}(X), \pm v_{1}(X), \pm v_{2}(X)\right)$ of $X$ to be $v(X)=v$.

To see that $v(X)$ is well defined (up to the ambiguity of sign in the last two entries), note that if $T^{\prime}$ also properly diagonalizes $X, \operatorname{Ad} T^{\prime}(X)=D\left(v^{\prime}\right)$, then $\Lambda=T^{\prime} T^{-1}$ transforms $D(v)$ to $D\left(v^{\prime}\right)$, and Lemma 5.2 applies. For a given ordered fixed point set $\left(q_{-}, q_{+}\right)$, there are in general four matrices $X$ with these fixed points and a given value of $v(X)$. We are now in a position to justify the following definition, which gives us an operation which modifies the invariant velocity while conserving the fixed points (recall the definitions at the beginning of this section).

Definition 5.5. Let $X \in \mathrm{sl}_{2} \mathbf{H}$ be strongly hyperbolic and let $c \in \mathbf{R}^{3}$. We define the product

$$
c X=\operatorname{Ad} T^{-1}(c \operatorname{Ad} T(X))
$$

where $T \in$ Aut $\widehat{\mathbf{H}}$ properly diagonalizes $X$.

Proposition 5.6. The product $c X$ is well defined, and the function $(c, X) \mapsto c X$ is a bilinear mapping for $c \in \mathbf{R}^{3}$ and strongly hyperbolic $X \in \mathrm{sl}_{2} \mathbf{H}$. For all $T \in$ Aut $\widehat{\mathbf{H}}$,

$$
c \operatorname{Ad} T(X)=\operatorname{Ad} T(c X) .
$$

Further, the invariant velocity $v$ satisfies the properties $v(c X)=c \cdot v(X)$ and $v(\operatorname{Ad} T(X))=v(X)$. 
Proof. Suppose $\operatorname{Ad} T(X)=D, \operatorname{Ad} T^{\prime}(X)=D^{\prime}$ where both $T, T^{\prime}$ properly diagonalize $X$. Then $\Lambda=T^{\prime} T^{-1}=\left(\lambda_{00}, 0 ; 0, \lambda_{11}\right)$ is a diagonal matrix and $D^{\prime}=\operatorname{Ad} \Lambda(D)$. Since $C_{\lambda_{00}}\left(v_{0}+v_{1} i\right)=v_{0}^{\prime}+v_{1}^{\prime} i$ it follows from Lemma 4.1 that $C_{\lambda_{00}}\left(c_{0} v_{0}+c_{1} v_{1} i\right)=$ $c_{0} v_{0}^{\prime}+c_{1} v_{1}^{\prime} i$; similarly $C_{\lambda_{11}}\left(-c_{0} v_{0}+c_{2} v_{2} i\right)=-c_{0} v_{0}^{\prime}+c_{2} v_{2}^{\prime} i$. Therefore $c D^{\prime}=$ Ad $\Lambda(c D)$; by this and (5.3) it follows that $c X$ is well defined. The remainder of the statement of the proposition follows directly from the definitions (5.2), (5.3).

In [19], the corresponding multiplication $c X$ for $X \in \mathrm{sl}_{2} \mathbf{C}$ was defined by letting $c \in \mathbf{C}$ and simply multiplying the entries of $X$ by $c$. By the commutativity of $\mathbf{C}$, this operation trivially commutes with $\operatorname{Ad} T$, so it corresponds to multiplying the diagonalized matrix by $c$. The definition we have given of $c X$ via (5.1), (5.2), (5.3) in $\mathrm{sl}_{2} \mathbf{H}$ unfortunately does not reduce, in the case of $\mathrm{sl}_{2} \mathbf{C}$, to that defined in [19]. It is tempting to remedy this by decomposing the diagonal $D(v)$ matrix in some other way, such as

$$
D(v)=\left(\begin{array}{cc}
-v_{0}+\frac{v_{1}-v_{2}}{2} i & 0 \\
0 & -v_{0}-\frac{v_{1}-v_{2}}{2} i
\end{array}\right)+\left(\begin{array}{cc}
\frac{v_{1}+v_{2}}{2} i & 0 \\
0 & \frac{v_{1}+v_{2}}{2} i
\end{array}\right),
$$

the unique representation as a sum of an element of $\mathrm{sl}_{2} \mathbf{C}$ and a diagonal matrix. One would like then to multiply the first summand by a complex number and the second by a real number. However, this would not respect the indeterminacy of signs in $v_{1}, v_{2}$ (recall Lemma 5.2) and thus would fail to introduce a well-defined notion of " $c X$ ".

The following condition will permit us later to "divide" by $X$ (cf. the proof of Theorem 5.11 below).

Definition 5.7. We say that $X$ is nonsingular if it is strongly hyperbolic and $v_{0}(X), v_{1}(X), v_{2}(X)$ are all nonzero.

Definition 5.8. An affine matrix $T=\left(t_{m n}\right) \in \mathrm{SL}_{2} \mathbf{H}$ (or its corresponding transformation $T \in$ Aff $\mathbf{H}$ ) is called semireal if $t_{00} \in \mathbf{R}$.

The following generalizes Proposition 2.10 in [19].

Lemma 5.9. Let $\left(p, p^{\prime}\right),\left(q, q^{\prime}\right) \in \mathbf{H}^{2}$ be pairs of distinct points. Then there exists a unique semireal affine $T \in$ Aut $\widehat{\mathbf{H}}$ such that $T(p)=q, T\left(p^{\prime}\right)=q^{\prime}$.

Proof. The hypothesis on $T$ requires $t_{10}=0$. The equations $t_{00} p+t_{01}=q t_{11}$, $t_{00} p^{\prime}+t_{01}=q^{\prime} t_{11}$ give $t_{00}\left(p-p^{\prime}\right)=\left(q-q^{\prime}\right) t_{11}$. Since $t_{00}$ is to be real and $\left|t_{00} t_{11}\right|=1$, we have $t_{00}^{2}=\left|q-q^{\prime}\right| /\left|p-p^{\prime}\right|$. This determines $\pm t_{00}$, and then $t_{11}, t_{01}$ are now found easily.

Definition 5.10. The strongly hyperbolic matrices $X, Y \in \mathrm{sl}_{2} \mathbf{H}$ are said to be completely similar if $Y=\operatorname{Ad} T(X)$ for some semireal affine $T$.

Theorem 5.11. Let $X \in \mathrm{sl}_{2} \mathbf{H}$ be nonsingular and nonaffine. Then there is a neighborhood of $X$ in which all elements are completely similar to $X$.

Proof. Let $T \in$ Aut $\widehat{\mathbf{H}}$ properly diagonalize $X, \operatorname{Ad} T(X)=D(v)$. Then there is a neighborhood of $X$ in which $v\left(X^{\prime}\right)=v^{\prime}$ satisfies $\left|v_{m}^{\prime}-v_{m}\right|<\left|v_{m}\right| / 2(m=0,1,2)$. This is because the fixed points of $X^{\prime}$ are close to those of $X$ by Lemma 4.6, so we can take $T^{\prime}$ close to $T$ with $\operatorname{Ad} T^{\prime}\left(X^{\prime}\right)=D\left(v^{\prime}\right)$, and make $v^{\prime}$ as close to $v$ as we like. Let $U$ be the unique semireal affine mapping, given by Lemma 5.9, taking the 
ordered fixed points of $X$ to those of $X^{\prime}$; thus $U$ is close to $I$. By the nonsingularity we can write $v^{\prime}=c \cdot v$ for suitable $c \in \mathbf{R}^{3}$ near $(1,1,1)$.

We claim that under the assumptions just made, $X^{\prime}=c \operatorname{Ad} U(X)$. To see this, note that by Proposition 5.6 this is the same as $c^{-1} \operatorname{Ad} U^{-1}\left(X^{\prime}\right)=X$, where we use the convenient notation $c^{-1}=\left(c_{0}^{-1}, c_{1}^{-1}, c_{2}^{-1}\right)$. Now, the two matrices $X$, $c^{-1} \operatorname{Ad} U^{-1}\left(X^{\prime}\right)$ have the same fixed point sets, and again by Proposition 5.6

$$
\begin{aligned}
v\left(c^{-1} \operatorname{Ad} U^{-1}\left(X^{\prime}\right)\right) & =c^{-1} v\left(\operatorname{Ad} U^{-1}\left(X^{\prime}\right)\right) \\
& =c^{-1} v\left(X^{\prime}\right) \\
& =c^{-1} v^{\prime}=v=v(X) .
\end{aligned}
$$

As remarked earlier, there are four matrices with the values $v(X)$, and one of them is $X$. Therefore if $c^{-1} \operatorname{Ad} U^{-1}\left(X^{\prime}\right)$ is sufficiently close to $X$, it is equal to $X$. This completes the proof.

\section{Contact of Curves with LOXodromes}

In [19] the notion of contact of a curve in $\widehat{\mathbf{C}}$ with a loxodrome was defined simply by equating the first three derivatives. Since $\mathrm{sl}_{2} \mathbf{C}$ and the space of 3-jets at a point share the same complex dimension, it was possible to find a correspondence between elements of the Lie algebra and nonsingular 3-jets. In Aut $\widehat{\mathbf{H}}$ the situation is quite different. The real dimension of $\mathrm{sl}_{2} \mathbf{H}$ is 15 , while a 3 -jet in $\widehat{\mathbf{H}}$ has 12 dimensions, insufficient to determine uniquely an element of $\operatorname{sl}_{2} \mathbf{H}$. It turns out that the situation is worse than this: a loxodromic trajectory is not even determined by its first four derivatives; we need to look at 5-jets, and deal with the fact that not every reasonable 5-jet of a curve in $\widehat{\mathbf{H}}$ need coincide with that of a loxodrome.

We begin by citing a basic result, the differential equation defining a loxodromic trajectory.

Proposition 6.1 ([21]). Let the loxodrome $\sigma$ be defined by (3.1). Then

$$
\dot{\sigma}=P_{X}(\sigma)
$$

where $P_{X}$ is defined by (4.2). Thus $\sigma$ is the unique solution of this differential equation with the initial condition $\sigma(0)=q$.

Given the $m$-jet $w=J_{m}(\gamma)=\left(\gamma ; \dot{\gamma}, \ddot{\gamma}, \ldots, \gamma^{(m)}\right)$ of a curve $\gamma$ and $T \in \operatorname{Aut} \widehat{\mathbf{H}}$, we will write $T_{*}(w)$ for the $m$-jet of the curve $T \circ \gamma$.

6.1. Schwarzian derivative. In [22] J. Ryan investigated the Schwarzian derivative of maps in Clifford algebras. For our purposes we will be concerned with Schwarzian derivatives of curves rather than of mappings. Let $\gamma:(-\varepsilon, \varepsilon) \rightarrow \widehat{\mathbf{H}}$ be a curve of class $C^{3}$.

Definition 6.2. The Schwarzian derivative of $\gamma$ is

$$
\mathcal{S}_{\gamma}=\dddot{\gamma}^{(3)} \dot{\gamma}^{-1}-(3 / 2)\left(\ddot{\gamma} \dot{\gamma}^{-1}\right)^{2} \text {. }
$$

There is an element of Aut $\widehat{\mathbf{H}}$ (not unique) which takes $\gamma(0)$ to 0 , so we will assume for the moment that $\gamma(0)=0$. We look for $T$ for which the curve $\tilde{\gamma}=T \circ \gamma$ satisfies

$$
\tilde{\gamma}(0)=0, \quad \dot{\tilde{\gamma}}(0)=1, \quad \ddot{\tilde{\gamma}}(0)=0
$$


i.e., $T_{*}\left(J_{2}(\gamma)(0)\right)=(0 ; 1,0)$. When this is carried out, it is seen that

$$
T=\left(\begin{array}{cc}
a & 0 \\
\frac{1}{2} a \ddot{\gamma} \dot{\gamma}^{-1} & a \dot{\gamma}
\end{array}\right)
$$

where $a \in \mathbf{H}-\{0\}$ is arbitrary (cf. [24]). The third derivative of $\tilde{\gamma}$ is found to be

$$
\tilde{\gamma}^{(3)}=a \mathcal{S}_{\gamma} a^{-1} \text {. }
$$

Finally, we take $a=\dot{\gamma}^{-1 / 2}$; this makes $T$ unique. (The quantity $\mathcal{S}_{\gamma}^{*}=\dot{\gamma}^{-1 / 2} \mathcal{S}_{\gamma} \dot{\gamma}^{1 / 2}$ $=\dot{\gamma}^{-1 / 2}\left(\sigma^{(3)}-(3 / 2) \ddot{\gamma} \dot{\gamma}^{-1} \ddot{\gamma}\right) \dot{\gamma}^{-1 / 2}$ would be in a sense a more natural definition of "Schwarzian derivative", as it does not favor multiplication on the left or right.) The above normalization operation of course can be carried out at any value of $t$ and does not require the simplifying assumption $\gamma(t)=0$.

6.2. Jets of loxodromic type. Before we can define the "loxodrome of closest contact" with a given curve, we must examine the nature of a loxodromic jet. Let $X \in \mathrm{sl}_{2} \mathbf{H}, q \in \mathbf{H}$, and consider the corresponding loxodrome $\sigma(t)=\exp (t X)(q)$. From Proposition 6.1 we obtain by induction that the derivatives of $\sigma$ are given by

$$
\begin{aligned}
\dot{\sigma} & =x_{00} \sigma-\sigma x_{11}-\sigma x_{10} \sigma+x_{01} \\
\sigma^{(n+1)} & =x_{00} \sigma^{(n)}-\sigma^{(n)} x_{11}-\sum_{m=0}^{n}\left(\begin{array}{c}
n \\
m
\end{array}\right) \sigma^{(m)} x_{10} \sigma^{(n-m)} \quad(n \geq 1) .
\end{aligned}
$$

We will assume that the loxodromic curve $\sigma$ has been normalized via (6.2), so that $q=\sigma(0)=0, \dot{\sigma}(0)=1, \ddot{\sigma}(0)=0$. Then at $t=0,(6.3)$ yields first

$$
x_{01}=1, x_{00}=x_{11}, \operatorname{Re} x_{00}=0
$$

(the last equality comes from $\operatorname{Re} \operatorname{tr} X=0$ ). From this,

$$
\begin{aligned}
& \sigma^{(3)}=-2 x_{10}, \\
& \sigma^{(4)}=2\left(x_{10} x_{00}-x_{00} x_{10}\right), \\
& \sigma^{(5)}=\left(x_{00} \sigma^{(4)}-\sigma^{(4)} x_{00}\right)+16 x_{10}^{2} .
\end{aligned}
$$

Thus we have computed the entries of the normalized 5 -jet

$$
J_{5}(\sigma)=\left(0 ; 1,0, \sigma^{(3)}, \sigma^{(4)}, \sigma^{(5)}\right)
$$

of $\sigma$. Recall that in particular, $\sigma^{(3)}$ is similar to $\mathcal{S}_{\sigma}^{*}$. Now we wish to invert the calculation, finding $X$ from the normalized 5 -jet of $\sigma$. If $x_{00}, x_{10}$ commute, then from (6.3) it follows that $\sigma^{(n)}$ is a monomial in $x_{10}$ for $n$ odd, and zero for $n$ even; then $\sigma$ lies in the plane $\mathbf{R}+\mathbf{R} x_{10}$. To avoid this degeneracy we will assume that $\sigma$ is nonplanar.

In what follows we will write $\mathbf{R}^{3}$ for the collection of quaternions with real part zero. Recall the classical vector operations $\langle a, b\rangle=(-1 / 2)(a b+b a), a \times b=$ $(1 / 2)(a b-b a)$ interrelated via quaternionic addition and multiplication, for any $a, b \in \mathbf{R}^{3}$. We will denote by $\vec{q}=\operatorname{Im} q=q-\operatorname{Re} q \in \mathbf{R}^{3}$ the purely vectorial part of a quaternion. Comparing with (6.5), write

$$
J_{5}(\sigma)=\left(0 ; 1,0, w_{3}, w_{4}, w_{5}+4 w_{3}^{2}\right) ;
$$

thus $w_{3}=-2 x_{10}$. Note that from (6.4), $x_{00} \in \mathbf{R}^{3}$. By (6.5), the quantities $w_{4}=2 x_{00} \times \vec{w}_{3}, w_{5}=2 x_{00} \times w_{4}$ are both in $\mathbf{R}^{3}$ as well, and satisfy the orthogonality relations

$$
x_{00} \perp w_{4}, x_{00} \perp w_{5}, \vec{w}_{3} \perp w_{4}, w_{4} \perp w_{5} .
$$


Since $\left|x_{00}\right|=\left|w_{5}\right| /\left(2\left|w_{4}\right|\right)$ and $x_{00}$ is orthogonal to $w_{4}$ and $w_{5}$, after checking the sign one deduces finally that $X$ is given in terms of $J_{5}(\sigma)$ by

$$
x_{00}=x_{11}=\frac{w_{4} \times w_{5}}{2\left|w_{4}\right|^{2}}, \quad x_{01}=1, \quad x_{10}=-w_{3} / 2 .
$$

We summarize the result as follows.

Theorem 6.3. Let $\sigma$ be a nonplanar loxodromic curve with the normalization $\sigma(0)=0, \dot{\sigma}(0)=1, \ddot{\sigma}(0)=0$. Then the 5 -jet of $\sigma$ is of the form (6.6) where $w_{3}, w_{4}, w_{5} \in \mathbf{H}$ satisfy the relations

$$
\operatorname{Re} w_{4}=\operatorname{Re} w_{5}=0, \quad \vec{w}_{3} \perp w_{4}, \quad w_{4} \perp w_{5} .
$$

Conversely, every 5 -jet of the form (6.6) with $w_{3}, w_{4} \notin \mathbf{R}$ and satisfying (6.8) is the 5-jet of a unique loxodromic trajectory $\sigma(t)=\exp (t X)(0)$ based at 0 , with $X$ given by (6.7).

6.3. Contact loxodromes. We will be interested in dealing with 5 -jets which are not necessarily of the special form treated in Proposition 6.3. Consider first an arbitrary normalized nonsingular 5 -jet, that is,

$$
\tilde{w}=\left(0 ; 1,0, \tilde{w}_{3}, \tilde{w}_{4}, \tilde{w}_{5}+4 \tilde{w}_{3}^{2}\right)
$$

where $\tilde{w}_{3}, \tilde{w}_{4}, \tilde{w}_{5} \in \mathbf{H}$ are arbitrary quaternions, subject only to the mild regularity condition $\tilde{w}_{3} \notin \mathbf{R}$. We will construct a loxodromic 5 -jet $\Psi(\tilde{w})$, which may be thought of as a "projection" of $\tilde{w}$ onto the class of jets described by (6.8). Let $w_{3}=\tilde{w}_{3}$, and then

$$
w_{4}=-\frac{\vec{w}_{3} \times\left(\vec{w}_{3} \times \overrightarrow{\tilde{w}}_{4}\right)}{\left|\vec{w}_{3}\right|^{2}}
$$

Thus $w_{4} \in \mathbf{R}^{3}$ and $\vec{w}_{3} \perp w_{4}$.

Now let

$$
w_{5}^{*}=\operatorname{Im}\left(\tilde{w}_{5}-4 w_{3}^{2}\right) \in \mathbf{R}^{3},
$$

and then

$$
w_{5}=-\frac{w_{4} \times\left(w_{4} \times w_{5}^{*}\right)}{\left|w_{4}\right|^{2}} .
$$

Thus $w_{5} \in \mathbf{R}^{3}$ and $w_{4} \perp w_{5}$. We will say that the normalized 5-jet $\tilde{w}$ is nonsingular if in the above construction, $\vec{w}_{3}$ and $w_{4}$ are not zero. A general 5-jet is called nonsingular if its normalized jet $T_{*}(w)$ is nonsingular.

Definition 6.4. Given $\tilde{w}_{3}, \tilde{w}_{4}, \tilde{w}_{5} \in \mathbf{H}$ with $\operatorname{Re} \tilde{w}_{3} \neq 0$, we define

$$
\Psi\left(\left(0 ; 1,0, \tilde{w}_{3}, \tilde{w}_{4}, \tilde{w}_{5}+4 \tilde{w}_{3}^{2}\right)\right)=\left(0 ; 1,0, w_{3}, w_{4}, w_{5}+4 w_{3}^{2}\right)
$$

as determined by $(6.9),(6.10),(6.11)$. For a general nonsingular 5 -jet, let us say $w=\left(q ; w_{1}, w_{2}, w_{3}, w_{4}, w_{5}\right)$, we choose $T \in$ Aut $\widehat{\mathbf{H}}$ so that the 2 -jet part of $T_{*} w$ is normalized, and define $\Psi(w)=\left(T^{-1}\right)_{*} \Psi\left(T_{*}(w)\right)$ where $\Psi\left(T_{*}(w)\right)$ is given by (6.12).

Note that in case $\operatorname{Re} \tilde{w}_{4}=0$ and $\overrightarrow{\tilde{w}}_{3} \perp \tilde{w}_{4}$ we have in fact $w_{4}=\tilde{w}_{4}$, and in case $\operatorname{Re} \tilde{w}_{5}=0$ and $w_{4} \perp w_{5}^{\prime}$, we have $w_{5}=\tilde{w}_{5}$.

It may be objected that some of the steps in the definition of $\Psi$ involve somewhat arbitrary choices. While this is true, the results of Section 6 will carry through for any choice of $\Psi$ for which the following fact is valid. 
Proposition 6.5. The smooth function $\Psi$ sends nonsingular 5 -jets to loxodromic 5-jets. If the 5-jet $w$ is loxodromic, then $\Psi(w)=w$. Further, $\Psi$ is Möbius invariant in the sense that $T_{*}(\Psi(w))=\Psi\left(T_{*}(w)\right)$ for all $T \in$ Aut $\widehat{\mathbf{H}}$.

From the preliminaries of this and the preceding sections, the following notions are well defined.

Definition 6.6. Let $\gamma:(-\varepsilon, \varepsilon) \rightarrow \mathbf{H}$ be a $C^{5}$-smooth curve. Assume that $J_{5}(\gamma)(t)$ is nonsingular for all $t$. The loxodrome enjoying contact with $\gamma$ at $\gamma(t)$ is $\kappa_{\gamma}(t)=$ $\left[A_{t}, X_{t}\right] \in \mathcal{B}$ where for each $t, \pi_{\mathcal{B}}\left(\kappa_{\gamma}(t)\right)=\gamma(t)$, and where the trajectory $\sigma$ of $\kappa_{\gamma}(t)$ satisfies $J_{5}(\sigma)(0)=\Psi\left(J_{5}(\gamma)(t)\right)$. If $\kappa_{\gamma}(t)$ is strongly hyperbolic we say that $\gamma$ is generic at $t$, and then the invariant velocity of $\gamma$ at $\gamma(t)$ is defined to be $v_{\gamma}=\left(v_{0}\left(X_{t}\right), \pm v_{1}\left(X_{t}\right), \pm v_{2}\left(X_{t}\right)\right)$.

By Proposition 6.5, we may extend the definition of contact and invariant velocity to curves passing through $\infty$ by applying first a transformation taking $\infty$ to a finite point.

\section{Quaternionic COVARIANT DERIVATIVE AND PARALLEL TRANSPORT}

7.1. Multiplication operators. Before defining the covariant derivative on $\mathcal{B}$, the bundle defined in (3.2), we introduce some elementary operations on loxodromic flow elements. The development follows formally the treatment in [19], but according to the definitions in the previous sections many of the concepts involved are quite different.

If $T \in$ Aut $\widehat{\mathbf{H}}$, then

$$
[A, X]=\left[A^{\prime}, X^{\prime}\right] \Rightarrow[T A, X]=\left[T A^{\prime}, X^{\prime}\right] .
$$

This furnishes an unambiguous definition of $T[A, X]=[T A, X]$, a germ whose trajectory is obtained by applying $T$ to that of $[A, X]$. By (7.1) and (3.3),

$$
\Phi_{T \alpha, t}(T \beta)=T \Phi_{\alpha, t}(\beta), \quad \alpha, \beta \in \mathcal{B} .
$$

If $\alpha=[A, X] \in \mathcal{B}$ is strongly hyperbolic and $(T, c) \in \operatorname{Aut} \widehat{\mathbf{H}} \times \mathbf{R}^{3}$, we define the generalized product

$$
(T, c) \alpha=(T, c)[A, X]=[T A, c X] \in \mathcal{B},
$$

where $c X$ is the bilinear operation of Definition 5.5. One should note that if $S \in$ Aff $\mathbf{H}$, then by Proposition 5.6, the relation

$$
(T, c)\left[A S^{-1}, \operatorname{Ad} S(X)\right]=\left[T A S^{-1}, \operatorname{Ad} S(c X)\right]
$$

holds, so (7.3) is well defined. We will say that the pair $\mu=(T, c)$ is a multiplier. Clearly $(T,(1,1,1)) \alpha=T \alpha,(I, c) \alpha=c \alpha=[A, c X]$.

Now let also $\beta=[B, Y]$ where $A(\infty)=B(\infty)=q$; i.e., $\pi_{B}(\alpha)=\pi_{B}(\beta)$. Suppose that $X$ and $Y$ are in fact nonsingular (recall that this implies strongly hyperbolic), and that they are related by $\operatorname{Ad} T(Y)=c X$. Define the quotient

$$
\frac{\beta}{\alpha}=\left(B T^{-1} A^{-1}, c\right) \in \operatorname{Aut} \widehat{\mathbf{H}} \times \mathbf{R}^{3} .
$$

By Proposition 5.11, such T, $c$ exist and are unique if $\beta$ is close to $\alpha$, so that at least in this case $\beta / \alpha$ is well defined. As would be expected,

$$
\frac{\beta}{\alpha} \alpha=\left(B T^{-1} A^{-1}, c\right)[A, X]=\left[B T^{-1}, c X\right]=\left[B T^{-1}, \operatorname{Ad} T(Y)\right]=[B, Y]=\beta .
$$


Similarly,

$$
\frac{T \alpha}{T \beta}=\operatorname{Ad} T \frac{\alpha}{\beta}
$$

where $\operatorname{Ad} T(\mu)$ is defined by conjugating the first component of $\mu$ by $T$.

Consider a smoothly parametrized family of multipliers $\mu_{t}=\left(T_{t}, c_{t}\right)$. Define its derivative to be the pair

$$
\frac{d \mu}{d t}=\left(\frac{d T}{d t}, \frac{d c}{d t}\right)
$$

Note that if $T_{0}=I$, then $\dot{T}=d T / d t \in \mathrm{sl}_{2} \mathbf{H}$ at $t=0$. Finally, define the exponential of an element of $\operatorname{sl}_{2} \mathbf{H} \times \mathbf{R}^{3}$ componentwise as $e^{(X, c)}=\left(e^{X}, e^{c}\right)$ where $e^{c}=\left(e^{c_{0}}, e^{c_{1}}, e^{c_{2}}\right)$.

Recall (3.4) that the loxodromic trajectory of $\alpha=[A, X]$ is

$$
\sigma_{\alpha}(t)=A\left(e^{t X}(\infty)\right)=A T^{-1}\left(e^{t \operatorname{Ad} T(X)}\right)(\infty)
$$

when $T \in$ Aff $\mathbf{H}$, which confirms that the trajectory is well defined independently of the representation of $\alpha$. If as above $\beta=[B, Y], B(\infty)=A(\infty)$, we define the primitive parallel transport $\Phi_{\alpha, t}(\beta)$ of $\beta$ along $\alpha$ for time $t$ as

$$
\Phi_{\alpha, t}(\beta)=\left[A e^{t X} A^{-1} B, Y\right]=\left[e^{t \operatorname{Ad} A(X)} B, Y\right],
$$

which is an element of $\mathcal{B}$ based at $A e^{t X} A^{-1} B(\infty)=A e^{t X}(\infty)=\sigma(t)$. Its trajectory is the $A$-image of the trajectory of $\beta$. Note that (3.3) is a special case, $\Phi_{\mathcal{B}}(\alpha, t)=$ $\Phi_{\alpha, t}(\alpha)$.

7.2. Covariant derivative. After these lengthy preliminaries, consider a family $\left\{\alpha_{t}\right\}$ in $\mathcal{B}$, and write $\alpha_{0}=\left[A_{0}, X_{0}\right]$. For the moment the values of $\alpha_{t}$ for $t \neq 0$ do not interest us; we want to differentiate along the trajectory of $\alpha_{0}$ a smooth family $\beta_{t}=\left[B_{t}, Y_{t}\right]$ based pointwise at $B_{t}(\infty)=\sigma(t)$, where $\sigma$ is the trajectory of $\alpha_{0}$; that is, $\pi_{\mathcal{B}}\left(\beta_{t}\right)=\sigma(t)$. Assume that $\alpha_{0}$ is nonsingular. Note that by (7.6), $\Phi_{\alpha_{0},-t}\left(\beta_{t}\right)$ is well defined and based at $\pi_{\mathcal{B}}\left(\alpha_{0}\right)=A_{0}(\infty)=\sigma(0)$. We also assume for the moment that this is not the point at infinity. By Theorem 5.11, for small $t, \Phi_{\alpha_{0},-t}\left(\beta_{t}\right)$ is completely similar to $\mathcal{B}_{0}$. Thus we can consider the quotient

$$
\frac{\Phi_{\alpha_{0},-t}\left(\beta_{t}\right)}{\beta_{0}} \in(\operatorname{Stab} q) \times \mathbf{R}^{3}
$$

where $\operatorname{Stab} q \subseteq$ Aut $\widehat{\mathbf{H}}$ is the stabilizer of $q$; it is a subgroup conjugate to Aff $\mathbf{H}$. Define

$$
\frac{d \beta}{d \alpha}(0)=\left.\frac{d}{d t}\right|_{t=0} \frac{\Phi_{\alpha_{0},-t}\left(\beta_{t}\right)}{\beta_{0}} \in \mathrm{sl}_{2} \mathbf{H} \times \mathbf{R}^{3}
$$

at $t=0$. For $t$ different from $0,(d \beta / d \alpha)(t)$ is defined analogously by means of an obvious change of variable.

Definition 7.1. The covariant derivative $D_{\alpha} \beta$ of $\left\{\beta_{t}\right\}$ along $\alpha$ at $t=0$ is

$$
D_{\alpha} \beta=e^{d \beta / d \alpha} \beta_{0} \in \mathcal{B}
$$

similarly it is defined for arbitrary $t$. 
This definition enjoys the following properties. First, $\pi_{\beta}\left(D_{\alpha} \beta\right)=\sigma(t)$, where $\sigma$ was defined at the beginning of this subsection. If for every $t, \beta_{t}$ happens to be the primitive parallel transport of $\beta_{0}$ along $\alpha$, i.e., $\beta_{t}=\Phi_{\alpha, t}\left(\beta_{0}\right)$, then $\Phi_{\alpha_{0},-t}\left(\beta_{t}\right) / \beta_{0}=$ $(I,(1,1,1))$, from which $d \beta / d \alpha=(0,(0,0,0))$ and then $D_{\alpha} \beta=(I,(1,1,1))$. These derivatives thus measure in some sense how much $\beta_{t}$ deviates locally from $\Phi_{\alpha_{0}, t}\left(\beta_{0}\right)$.

Let $T \in$ Aut $\widehat{\mathbf{H}}$. Then by (7.2), (7.5),

$$
\frac{d(T \beta)}{d(T \alpha)}=\operatorname{Ad} T\left(\frac{d \beta}{d \alpha}\right),
$$

from which we have the following important Möbius invariance.

Lemma 7.2. Let $\alpha_{0}, \beta_{t}$ in $\mathcal{B}$ be as described, and let $T \in$ Aut $\widehat{\mathbf{H}}$. Then

$$
D_{T \alpha} T \beta=T D_{\alpha} \beta .
$$

This lemma permits us to remove the restriction that $\pi_{\mathcal{B}}\left(\alpha_{0}\right) \neq \infty$ in defining $D_{\alpha} \beta$.

We now express the covariant derivative $D_{\alpha} \beta$ explicitly in terms of $A, B, X$, $Y$. Assume that $B_{t}, Y_{t}$ vary smoothly with $t$. Take $T_{t} \in$ Aut $\widehat{\mathbf{H}}, y_{t} \in \mathbf{R}^{3}$ so that $\operatorname{Ad} T_{t}\left(Y_{t}\right)=y_{t} Y_{0}$. The proof of Lemma 5.9 shows that $T_{t}$ depends smoothly on $t$, and then so does $y_{t}$. We have $\beta_{t}=\left[B_{t} T_{t}^{-1}, y_{t} Y_{0}\right], T_{0}=I, y_{0}=(1,1,1)$. Now we can expand

$$
\begin{aligned}
\frac{d \beta}{d \alpha} & =\left.\frac{d}{d t}\right|_{t=0}\left(A e^{-t X} A^{-1} B_{t} T_{t}^{-1} B_{0}^{-1}, y_{t}\right) \\
& =\left(-\operatorname{Ad} A(X)+\dot{B}_{0} B_{0}^{-1}-\dot{T}, \dot{y}_{0}\right)
\end{aligned}
$$

where the dots indicate $t$-derivatives. Then

$$
D_{\alpha} \beta=\left(e^{\operatorname{Ad} A(X)+\dot{B}_{0} B_{0}^{-1}-\dot{T}}, e^{\dot{y}_{0}}\right) .
$$

If $T_{t}=I$ for all $t$, then $\dot{T}=0$. We say that $\beta_{t}, Y_{t}$ are normalized representatives for $\beta$ when this occurs; such representatives always exist because of the definition $(3.2)$.

7.3. Parallel transport along a smooth curve. We have defined primitive parallel transport along a loxodromic trajectory. Now we extend this concept to transport elements of $\mathcal{B}$ along any generic curve. Recall the contact loxodrome $\kappa_{\gamma}$ of Definition 6.6.

Definition 7.3. Let $\gamma:(-\varepsilon, \varepsilon) \rightarrow \widehat{\mathbf{H}}$ be a generic smooth curve. Let $\beta:(-\varepsilon, \varepsilon) \rightarrow$ $\mathcal{B}$ be smooth, satisfying $\pi_{B}\left(\beta_{t}\right)=\gamma(t)$. Then define $d \beta / d \gamma, D_{\gamma} \beta$ by

$$
\frac{d \beta}{d \gamma}=\frac{d \beta}{d \kappa_{\gamma}}, \quad D_{\gamma} \beta=D_{\kappa_{\gamma}} \beta
$$

where $\kappa_{\gamma}$ is the contact loxodrome to $\gamma$ given in Definition 6.6.

Definition 7.4. Let $\gamma, \beta$ be as above. Then $\beta$ is parallel along $\gamma$ if $d \beta / d \gamma=$ $(0,(0,0,0))$. We then say that $\beta_{t}$ is the parallel transport of $\beta_{0}$ along $\gamma$.

Note that if $\beta$ is parallel along $\gamma$, then $D_{\gamma} \beta=\beta$ since $\exp \left(d \beta / d \kappa_{\gamma}\right)=(I,(1,1,1))$. It remains to show that "the" parallel transport is well defined; the proof below is essentially the same as that of [19]. 
Theorem 7.5. Let $\gamma:(-\varepsilon, \varepsilon) \rightarrow \widehat{\mathbf{H}}$ be smooth and generic, and suppose $\beta_{0} \in \mathcal{B}$, $\pi_{B}\left(\beta_{0}\right)=\gamma(0)$. Then there exists a unique family $\beta:(-\varepsilon, \varepsilon) \rightarrow \mathcal{B}$ such that $\beta_{t}=\beta_{0}$ for $t=0, \pi_{\mathcal{B}}\left(\beta_{t}\right)=\gamma(t)$, and such that $\beta$ is parallel along $\gamma$.

Proof. By Lemma 7.2 we may assume $\gamma(0) \in \mathbf{H}$. Write $\beta_{0}=\left[B_{0}, Y_{0}\right]$, and let $\alpha_{t}=\kappa_{\gamma}(t)=\left[A_{t}, X_{t}\right]$. Consider the ordinary differential equation

$$
\dot{B}=A X A^{-1} B,
$$

which has a unique solution $B_{t}$ with initial condition $B_{0}$ at $t=0$. This permits us to define $\beta_{t}=\left[B_{t}, Y_{0}\right]$. We claim that

$$
\pi_{\mathcal{B}}\left(\beta_{t}\right)=\pi_{\mathcal{B}}\left(\alpha_{t}\right) \text {. }
$$

Once this is verified, then it follows by (7.10), (7.12) that $\left\{\beta_{t}\right\}$ is parallel along $\gamma$. Now (7.13) holds for $t=0$, since

$$
B_{0}(\infty)=A_{0}(\infty) \neq \infty .
$$

The following can be verified by elementary calculus.

Lemma 7.6. Let $B_{t}, B_{t}^{\prime} \in \mathrm{SL}_{2} \mathbf{H}, B_{0}(\gamma(0))=B_{0}^{\prime}(\gamma(0)) \neq \infty, B_{t}^{\prime}=B_{t}+\mathcal{O}\left(t^{2}\right)$ as $t \rightarrow 0$. Then $B_{t}^{\prime}(\gamma(0))=B_{t}(\gamma(0))+\mathcal{O}\left(t^{2}\right)$.

From this lemma we have

$$
B_{t} B_{0}^{-1}(\gamma(0))=e^{t \dot{B}_{0} B_{0}^{-1}}(\gamma(0))+\mathcal{O}\left(t^{2}\right) .
$$

By (7.12), $e^{t \dot{B}_{0} B_{0}^{-1}}=A_{0} e^{t X_{0}} A_{0}^{-1}$ for all $t$, so $B_{t}(\infty)=A_{0} e^{t X_{0}}(\infty)+\mathcal{O}\left(t^{2}\right)$. This says

$$
\begin{aligned}
\left.\frac{d}{d t}\right|_{t=0} B_{t}(\infty) & =\left.\frac{d}{d t}\right|_{t=0} A_{0} e^{t X_{0}}(\infty) \\
& =\left.\frac{d}{d t}\right|_{t=0} A_{t}(\infty)
\end{aligned}
$$

because of the contact relation $\alpha=\kappa_{\gamma}$. Adjusting the notation so that what we have shown for $t=0$ is reflected for general $t$, this becomes

$$
\frac{d}{d t} B_{t}(\infty)=\frac{d}{d t} A_{t}(\infty)=\frac{d \gamma(t)}{d t}
$$

Combining this with (7.14), we conclude that $B_{t}(\infty)=\gamma(t)$ for all $t$; i.e., (7.13) is valid as claimed. Thus the parallel transport $\beta$ exists. The uniqueness follows similarly from the theory of ordinary differential equations.

The covariant derivative provides a characterization of loxodromes, justifying the statement that loxodromes act like "geodesics" in Möbius geometry:

Proposition 7.7. A self-parallel curve in $\widehat{\mathbf{H}}$ is loxodromic: if $\kappa_{\gamma}$ is parallel along $\gamma$, then $\gamma$ is a loxodromic trajectory.

Proof. Suppose $D_{\gamma} \kappa_{\gamma}=0$. Take normalized representatives $B_{t}, Y_{t}=y_{t} Y_{0}$ for $\beta=$ $\kappa_{\gamma}$. Then by (7.10) the hypothesis $d \beta / d \beta=0$ implies $\dot{y}=0$, so $y_{t}=y_{0}=(1,1,1)$. Then $Y_{t}=Y_{0}$ for all $t$. Now (7.12) can be written $\dot{B} B^{-1}=\operatorname{Ad} B(Y)$, so

$$
\dot{B}=B Y_{0} .
$$

Then $\beta_{t}=B_{0} \exp \left(t Y_{0}\right)$, so $\gamma$ is a loxodrome. 


\section{Closing observations}

We wish to stress two points which are important for understanding the geometry of the quaternionic covariant derivative.

For Aut $\widehat{\mathbf{C}}$, there is a formulation of Proposition 7.7 which makes no mention of the covariant derivative, namely, that a curve whose invariant velocity is constant is a loxodrome [19]. This is connected with the fact that the invariant velocity of a curve in $\widehat{\mathbf{C}}$ is equal to its Schwarzian derivative. In $\widehat{\mathbf{H}}$ the situation is different. The Schwarzian derivative of a loxodrome is not, in general, constant. Consider for example the spiral $\sigma(t)=e^{t X}(1)=e^{t u} e^{-t v}$ based at 1 where $X=(u, 0 ; 0, v)$ and $u, v$ do not commute. One sees that $\mathcal{S}_{\sigma}(t)=e^{t u} R(u, v) e^{-t u}$ where $R(u, v)$ is a rational expression in $u, v$, constant with respect to $t$; it is easy to see that in general $R(u, v)$ does not commute with $e^{t u}$. Similarly it can be seen that $\mathcal{S}_{\sigma}^{*}$ is not constant.

It is not apparent what the relation is between the three dimensional invariant velocity $v(X)$ and the four dimensional Schwarzian derivative $\mathcal{S}_{\sigma}$. This question merits further investigation.

The second point relates to the fixed points of strongly hyperbolic transformations. Let $\gamma$ be a nonsingular smooth curve in the sense of Section 5 . Then the contact loxodrome $\kappa_{\gamma}(t)=\left[A_{t}, X_{t}\right]$ to $\gamma$ possesses a fixed point set $\left(q_{-, \gamma}(t), q_{+, \gamma}(t)\right)$. We have seen that these depend smoothly on $t$. Since the fixed points are determined by the entries of $A_{t}, X_{t}$, and these in turn are determined (with suitable normalizations) by the 5 -jet of $\gamma$, there must exist smooth functions $Q_{-}, Q_{+}$defined on the space of nonsingular 5-jets, satisfying

$$
Q_{-}\left(J_{5}(\gamma)(t)\right)=q_{-, \gamma}(t), \quad Q_{+}\left(J_{5}(\gamma)(t)\right)=q_{+, \gamma}(t)
$$

for all $\gamma$, and enjoying the Möbius invariance property

$$
Q_{-}\left(J_{5}(T \circ \gamma)(t)\right)=T\left(q_{-, \gamma}(t)\right), \quad Q_{+}\left(J_{5}(T \circ \gamma)(t)\right)=T\left(q_{+, \gamma}(t)\right) .
$$

As was remarked earlier, the calculation of fixed points in Aut $\widehat{\mathbf{H}}$ reduces to an intractable quadratic equation, so $Q_{+}, Q_{-}$are not known explicitly. In contrast, in $\widehat{\mathbf{C}}$ it is of course simple to calculate the fixed points of a Möbius transformation. This leads to the following Möbius invariance, given in [19].

Proposition 8.1. Let $z=z(t)$ be a $C^{3}$ curve in the Riemann sphere $\widehat{\mathbf{C}}$. Let $T \in$ Aut $\widehat{\mathbf{C}}$ be fixed, and let $w(t)=T(z(t))$. Then

$$
T\left(z-\frac{2 \dot{z}^{2}}{\ddot{z} \pm \dot{z} \sqrt{-2 \mathcal{S}_{z}}}\right)=w-\frac{2 \dot{w}^{2}}{\ddot{w} \pm \dot{w} \sqrt{-2 \mathcal{S}_{w}}} .
$$

It would therefore be of great interest to know more about the nature of the quaternionic functions $Q_{ \pm}$.

\section{REFERENCES}

1. Ahlfors, L. V., Old and new in Möbius groups, Ann. Acad. Sci. Fenn. Ser. A I Math. 9 (1984), 93-105. MR 86b:22018

2. Clifford numbers and Möbius transformations in $\mathbf{R}^{n}$, in Clifford Algebras and their Applications in Mathematical Physics, J. S. R. Chrisholm and A. K. Common, eds., Nato Adv. Study Inst. Ser., Ser. C: Math. Phys. Sci. 183 (1986), 167-175. MR 88b:20074

3. Aslaksen, H., Quaternionic determinants, Math. Intelligencer 18 (1996), 57-65. MR 97j:16028 
4. Beardon, A., The Geometry of Discrete Groups, Springer-Verlag, New York (1983). MR 85d:22026; MR 97d:22011

5. Brackx, F., Delanghe, R., and Sommen, F., Clifford Analysis, Pitman (1982). MR 85j:30103

6. Cnops, J., Spherical geometry and Möbius transformations, in Clifford Algebras and their Applications in Mathematical Physics, Dienze, 1993, ed. Brackx, F.; Delanghe, R.; and Serras, H., Kluwer Academic Publishers (1993). MR 95e:53022

7. Greenberg, P. and Porter, R. M., A generalization of geodesic flow, Aportaciones Matemáticas, Comunicaciones 4 (1987), 197-204. MR 90c:58141

8. Gunning, R. C., On uniformization of manifolds: the role of connections, Princeton University Press, Princeton (1978). MR 82e:32034

9. Harvey, F. R., Spinors and Calibrations, Academic Press (1990). MR 91e:53056

10. Heidrich, R. and Jank, G., On the iteration of quaternionic Möbius transformations, Complex Variables Theory Appl. 29 (1996), 313-318. MR 97e:30079

11. Hellegouarch, Y., Quaternionic homographies: application to Ford hyperspheres, C. R. Math. Rep. Acad. Sci. Canada 11 (1989), 171-176. MR 91j:11023

12. Kähler, E., Die Poincaré-Gruppe, Rend. Sem. Mat. Fis. Milano (1983) 53 (1986), 359-390. MR 87m:22059

13. Koecher, M. and Remmert, R., Hamilton's quaternions, in J. H. Ewing, ed., Numbers, Graduate Texts in Mathematics 123, Springer-Verlag, New York (1991), 189-220.

14. Kra, I., Quadratic differentials, Rev. Roumaine Math. Pures Appl. 39 (1994), 751-787. MR 95m:30058

15. Kra, I. and Maskit, B., Remarks on projective structures, Riemann Surfaces and Related Topics: Proceedings of the 1978 Stony Brook Conference, ed. I. Kra and B. Maskit, Ann. of Math. Studies, Princeton Univ. Press, Princeton, NJ (1981), 343-359. MR 83f:30042

16. Lounesto, P. and Springer, A., Möbius transformations and Clifford algebras of Euclidean and anti-Euclidean spaces, Deformations of mathematical structures (Eódź/Lublin, 1985/87), Kluwer Acad. Publ., Dordrecht (1989), 79-90. MR 90d:30131

17. Lounesto, P. and Latvamaa, E., Conformal transformations and Clifford algebras, Proc. Amer. Math. Soc. 79 (1980), 533-538. MR 81h:15017

18. Niven, I., Equations in quaternions, Amer. Math. Monthly 48 (1941), 654-661. MR 3:264b

19. Porter, R. M., Differential invariants in Möbius geometry, J. Natural Geometry 3 (1993), 97-123. MR 94d:30077

20. _ Quaternionic linear and quadratic equations, J. Natural Geometry 11 (1997), 101106. MR 98b:15030

21. __ Quaternionic Möbius transformations and loxodromes, Complex Variables, Theory and Applications (to appear).

22. Ryan, J., Generalized Schwarzian derivatives for generalized fractional linear transformations, Ann. Polon. Math. 57 (1992), 29-44. MR 94f:30062

23. Shub, M., Global Stability of Dynamical Systems, Springer-Verlag, New York (1987).

24. Thurston, W., Zippers and univalent functions, The Bieberbach conjecture: Proceedings of the symposium on the occasion of the proof, Math. Surveys Monogr. 21, Amer. Math. Soc., Providence, RI, 1986, pp. 185-197. MR 88j:30040

25. Varadarajan, V. S., Lie groups, Lie Algebras and Their Representations, Springer-Verlag, New York (1984). MR 85e:22001

26. Wada, M., Conjugacy invariants of Möbius transformations, Complex Variables Theory Appl. 15 (1990), 125-133. MR 92a:30048

Departamento de Matemáticas, Centro de Investigación y de Estudios Avanzados del I.P.N., Apdo. Postal 14-740, 07000 México D. F., Mexico

E-mail address: mike@math.cinvestav.mx 